\title{
Article
}

\section{On Leonardo Pisano Hybrinomials}

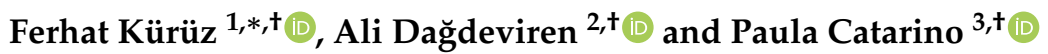 \\ 1 Department of Computer Engineering, Istanbul Gelisim University, Istanbul 34310, Turkey \\ 2 Department of Weight and Balance, Turkish Aviation Academy, Istanbul 34149, Turkey; \\ m.a.dagdeviren@gmail.com \\ 3 Department of Mathematics, University of Trás-os-Montes and Alto Douro, Quinta de Prados, \\ 5001-801 Vila Real, Portugal; pcatarino23@gmail.com \\ * Correspondence: fkuruz@gelisim.edu.tr \\ + These authors contributed equally to this work.
}

check for

updates

Citation: Kürüz, F.; Dağdeviren, A.; Catarino, P. On Leonardo Pisano Hybrinomials. Mathematics 2021, 9, 2923. https://doi.org/10.3390/ math 9222923

\section{Academic Editors: Araceli}

Queiruga-Dios, Maria Jesus Santos, Fatih Yilmaz, Deolinda M. L. Dias

Rasteiro, Jesús Martín Vaquero and Víctor Gayoso Martínez

Received: 17 October 2021

Accepted: 15 November 2021

Published: 17 November 2021

Publisher's Note: MDPI stays neutral with regard to jurisdictional claims in published maps and institutional affiliations.

Copyright: (C) 2021 by the authors Licensee MDPI, Basel, Switzerland. This article is an open access article distributed under the terms and conditions of the Creative Commons Attribution (CC BY) license (https:/ / creativecommons.org/licenses/by/ $4.0 /)$.
Abstract: A generalization of complex, dual, and hyperbolic numbers has recently been defined as hybrid numbers. In this study, using the Leonardo Pisano numbers and hybrid numbers we investigate Leonardo Pisano polynomials and hybrinomials. Furthermore, we also describe the basic algebraic properties and some identities of the Leonardo Pisano polynomials and hybrinomials.

Keywords: hybrid numbers; Leonardo Pisano numbers; hybrinomials

\section{Introduction}

Fibonacci numbers, for $n \geq 2$, are given by the recurrence relation $F_{n}=F_{n-1}+F_{n-2}$ with the initial conditions $F_{0}=0, F_{1}=1$. There are many works regarding Fibonacci numbers, such as [1-3]. Furthermore, they have also been studied on different number systems such as quaternions and hybrid numbers $[4,5]$.

When the literature is examined, it is seen that Leonardo Pisano numbers are only called Leonardo numbers. However, the name Leonardo in these studies refers to Leonardo Pisano, who is also called Fibonacci, from the Latin Filius Bonacci. It is well known that Leonardo commonly stands for Leonardo Da Vinci, so in this study to avoid confusion we prefer to use the name "Leonardo Pisano".

Leonardo Pisano numbers have recently been studied in detail by Catarino and Borges in [6] and they have demonstrated some properties of this sequence. For $n \geq 2$, Leonardo Pisano numbers are defined by the following recurrence relation

$$
L e_{n}=L e_{n-1}+L e_{n-2}+1
$$

where the initial conditions are $L e_{0}=L e_{1}=1$. Moreover, another recurrence relation for Leonardo Pisano's numbers is

$$
L e_{n+1}=2 L e_{n}-L e_{n-2} .
$$

The relationship between Leonardo Pisano numbers and Fibonacci numbers can be given as

$$
L e_{n}=2 F_{n+1}-1
$$

Binet's formula and the characteristic equation of Leonardo Pisano numbers are

$$
L e_{n}=\frac{2 \alpha^{n+1}-2 \beta^{n+1}-\alpha+\beta}{\alpha-\beta} \text { and } \lambda^{3}-2 \lambda^{2}+1=0
$$

respectively. Cassini's, Catalan and d'Ocagne's identities were defined in [6]. In [7] generalized Leonardo Pisano numbers have been defined. In [8], incomplete Leonardo Pisano numbers and matrix representation of Leonardo Pisano numbers have also been 
given. Additionally, in [9] the authors give some important properties of Leonardo Pisano numbers and in [10] hybrid Leonardo Pisano numbers are defined.

Hybrid numbers are a generalization of the complex, dual and hyperbolic numbers. A hybrid number can be denoted by $w=r_{0}+r_{1} \mathbf{i}+r_{2} \varepsilon+r_{3} \mathbf{h}$, where $r_{0}, r_{1}, r_{2}, r_{3}$ are real numbers [11]. Hybrid numbers, $\mathbb{K}$, are defined as

$$
\mathbb{K}=\left\{w=r_{0}+r_{1} \mathbf{i}+r_{2} \varepsilon+r_{3} \mathbf{h}: r_{0}, r_{1}, r_{2}, r_{3} \in \mathbb{R}, \begin{array}{c}
\mathbf{i}^{2}=-1, \varepsilon^{2}=0, \mathbf{h}^{2}=1 \\
\mathbf{i h}=-\mathbf{h i}=\boldsymbol{\varepsilon}+\mathbf{i}
\end{array}\right\} .
$$

Equality, addition and multiplication are defined by

(i) $w_{1}=w_{2} \Longleftrightarrow r_{0}=s_{0}, r_{1}=s_{1}, r_{2}=s_{2}, r_{3}=s_{3}$,

(ii) $w_{1}+w_{2}=\left(r_{0}+s_{0}\right)+\left(r_{1}+s_{1}\right) \mathbf{i}+\left(r_{2}+s_{2}\right) \boldsymbol{\varepsilon}+\left(r_{3}+s_{3}\right) \mathbf{h}$,

(iii) $w_{1} \cdot w_{2}=\left(r_{0}+r_{1} \mathbf{i}+r_{2} \varepsilon+r_{3} \mathbf{h}\right) \cdot\left(s_{0}+s_{1} \mathbf{i}+s_{2} \varepsilon+s_{3} \mathbf{h}\right)$

$$
\begin{aligned}
= & \left(r_{0} s_{0}-r_{1} s_{1}+r_{2} s_{1}-r_{1} s_{2}+r_{3} s_{3}\right)+\left(r_{0} s_{2}+r_{1} s_{0}+r_{1} s_{3}-r_{3} s_{1}\right) \mathbf{i} \\
& +\left(r_{0} s_{2}+r_{2} s_{0}-r_{2} s_{3}+r_{3} s_{2}+r_{1} s_{3}-r_{3} s_{1}\right) \varepsilon+\left(r_{0} s_{3}+r_{3} s_{0}+r_{1} s_{2}+r_{2} s_{1}\right) \mathbf{h},
\end{aligned}
$$

for hybrid numbers $w_{1}=r_{0}+r_{1} \mathbf{i}+r_{2} \boldsymbol{\varepsilon}+r_{3} \mathbf{h}$ and $w_{2}=s_{0}+s_{1} \mathbf{i}+s_{2} \boldsymbol{\varepsilon}+s_{3} \mathbf{h}$. More details on hybrid numbers can be found in [11].

Fibonacci polynomials were first studied by Bicknell [12]. Since then, many authors have published works on the subject of Fibonacci-type polynomials [13-17]. These types of polynomials have some considerable applications in number theory, geometry and algebra. Moreover, hybrinomial sequences have become an increasing area of attention. For instance, the authors studied Fibonacci and Lucas hybrinomials [18], Petroudi and Pirouz studied Van der Laan hybrinomials [19] and Petroudi et al. studied Narayana polynomials and Narayana hybrinomial sequences [20]. Moreover, Horadam hybrinomials were studied by Kızılateş in [21].

In this work, in Section 2, we define the Leonardo Pisano polynomials and hybrinomials. Then, in Section 3, we present some identities of Leonardo Pisano polynomial and hybrinomial sequences such as Catalan-like identities and summing formulas.

\section{Leonardo Pisano Polynomials and Hybrinomials}

In this section, we introduce Leonardo Pisano polynomials and hybrinomials.

Definition 1. For a non-negative integer $n$, Leonardo Pisano polynomials $L e_{n}(x)$ are defined by $\operatorname{Le}_{n}(x)=\left\{\begin{array}{ll}1 & , n=0,1 \\ x+2 & , n=2 \\ 2 x L e_{n-1}(x)-L e_{n-3}(x) & , n \geq 3\end{array}\right.$.

The first few Leonardo Pisano polynomials are $1,1, x+2,2 x^{2}+4 x-1,4 x^{3}+8 x^{2}-$ $2 x-1,8 x^{4}+16 x^{3}-4 x^{2}-3 x-2,16 x^{5}+32 x^{4}-8 x^{3}-8 x^{2}-8 x+1$. Using Leonardo Pisano polynomials, we can define Leonardo Pisano hybrinomials.

Definition 2. The nth Leonardo Pisano hybrinomial is defined as follows:

$$
L e_{n}^{[H]}(x)=L e_{n}(x)+\mathbf{i} L e_{n+1}(x)+\varepsilon L e_{n+2}(x)+\mathbf{h} L e_{n+3}(x) .
$$

The first four Leonardo Pisano hybrinomials are 


$$
\begin{aligned}
L e_{0}^{[H]}(x)= & 1+\mathbf{i}+\varepsilon(x+2)+\mathbf{h}\left(2 x^{2}+4 x-1\right) \\
L e_{1}^{[H]}(x)= & 1+\mathbf{i}(x+2)+\varepsilon\left(2 x^{2}+4 x-1\right)+\mathbf{h}\left(4 x^{3}+8 x^{2}-2 x-1\right) \\
L e_{2}^{[H]}(x)= & (x+2)+\mathbf{i}\left(2 x^{2}+4 x-1\right)+\varepsilon\left(4 x^{3}+8 x^{2}-2 x-1\right) \\
& +\mathbf{h}\left(8 x^{4}+16 x^{3}-4 x^{2}-3 x-2\right) \\
L e_{3}^{[H]}(x)= & \left(2 x^{2}+4 x-1\right)+\mathbf{i}\left(4 x^{3}+8 x^{2}-2 x-1\right)+\varepsilon\left(8 x^{4}+16 x^{3}-4 x^{2}-3 x-2\right) \\
& +\mathbf{h}\left(16 x^{5}+32 x^{4}-8 x^{3}-8 x^{2}-8 x+1\right) .
\end{aligned}
$$

If we put $x=1$, then we obtain

$$
\begin{aligned}
& L e_{0}^{[H]}(x)=1+\mathbf{i}+3 \boldsymbol{\varepsilon}+5 \mathbf{h}=\mathrm{LeH}_{0} \\
& L e_{1}^{[H]}(x)=1+3 \mathbf{i}+5 \boldsymbol{\varepsilon}+9 \mathbf{h}=\mathrm{LeH}_{1} \\
& L e_{2}^{[H]}(x)=3+5 \mathbf{i}+9 \boldsymbol{\varepsilon}+15 \mathbf{h}=\mathrm{LeH}_{2} \\
& L e_{3}^{[H]}(x)=5+9 \mathbf{i}+15 \boldsymbol{\varepsilon}+25 \mathbf{h}=\mathrm{LeH}_{3}
\end{aligned}
$$

where $\mathrm{LeH}_{n}$ stand for Leonardo Pisano hybrid numbers. Therefore, it can be easily seen that Leonardo Pisano hybrinomials are a generalization of Leonardo Pisano hybrid numbers.

We can define the character of Leonardo Pisano hybrinomials by making use of the character of hybrid numbers that Özdemir defined in his article.

Definition 3. The character of Leonardo Pisano hybrinomials is

$$
C\left(L e_{n}^{[H]}(x)\right)=L e_{n}^{2}(x)+L e_{n+1}^{2}(x)-2 L e_{n+1}(x) L e_{n+2}(x)-L e_{n+3}^{2}(x) .
$$

Using the character of the Leonardo Pisano hybrinomials, we can set the norm.

Theorem 1. The norm of the Leonardo Pisano hybrinomials $L e_{n}^{[H]}(x)$ is given by

$$
\left\|L e_{n}^{[H]}(x)\right\|=\sqrt{\left|L e_{n+1}^{2}(x)-2 L e_{n+1}(x) L e_{n+2}(x)-4 x^{2} L e_{n+2}^{2}(x)+4 x L e_{n+2}(x) L e_{n}(x)\right|} .
$$

Proof. From the character of Leonardo Pisano hybrinomials which is given above,

$$
\begin{aligned}
& \left\|L e_{n}^{[H]}(x)\right\|^{2} \\
& =\left|L e_{n}^{2}(x)+L e_{n+1}^{2}(x)-2 L e_{n+1}(x) L e_{n+2}(x)-L e_{n+3}^{2}(x)\right| \\
& =\left|L e_{n}^{2}(x)+L e_{n+1}(x)-2 L e_{n+1}(x) L e_{n+2}(x)-\left(2 x L e_{n+2}(x)-L e_{n}(x)\right)^{2}\right| \\
& =\left|L e_{n+1}^{2}(x)-2 L e_{n+1}(x) L e_{n+2}(x)-4 x^{2} L e_{n+2}^{2}(x)+4 x L e_{n+2}(x) L e_{n}(x)\right| .
\end{aligned}
$$

In the following theorem using the matrix representation of hybrid numbers:

$$
M(a+b \mathbf{i}+c \mathcal{E}+d \mathbf{h})=\left[\begin{array}{cc}
a+c & b-c+d \\
c-b+d & a-c
\end{array}\right]
$$

we give the matrix representation for Leonardo Pisano hybrinomials.

Theorem 2. Every Leonardo Pisano hybrinomials can be written in the following matrix form, for $L e_{m}^{[H]}(x)$

$$
M\left(L e_{m}^{[H]}(x)\right)=\left[\begin{array}{cc}
L e_{m}(x)+L e_{m+2}(x) & L e_{m+1}(x)-L e_{m+2}(x)+L e_{m+3}(x) \\
L e_{m+2}(x)+L e_{m+3}(x)-L e_{m+1}(x) & L e_{m}(x)-L e_{m+2}(x)
\end{array}\right]
$$


Proof. Using the matrix equivalent of hybrid units $1, \mathbf{i}, \varepsilon$ and $\mathbf{h}$, we have

$$
\begin{aligned}
& L e_{m}^{[H]}(x)=L e_{m}(x)+\mathbf{i} L e_{m+1}(x)+\varepsilon L e_{m+2}(x)+\mathbf{h} L e_{m+3}(x) \\
& M\left(L e_{m}^{[H]}(x)\right)=L e_{m}(x)\left[\begin{array}{ll}
1 & 0 \\
0 & 1
\end{array}\right]+L e_{m+1}(x)\left[\begin{array}{cc}
0 & 1 \\
-1 & 0
\end{array}\right]+L e_{m+2}(x)\left[\begin{array}{ll}
1 & -1 \\
1 & -1
\end{array}\right] \\
& +L e_{m+3}(x)\left[\begin{array}{ll}
0 & 1 \\
1 & 0
\end{array}\right]
\end{aligned}
$$

then we can write

$$
M\left(L e_{m}^{[H]}(x)\right)=\left[\begin{array}{cc}
L e_{m}(x)+L e_{m+2}(x) & L e_{m+1}(x)-L e_{m+2}(x)+L e_{m+3}(x) \\
L e_{m+2}(x)+L e_{m+3}(x)-L e_{m+1}(x) & L e_{m}(x)-L e_{m+2}(x)
\end{array}\right]
$$

Lemma 1. Let $L e_{n}^{[H]}(x)$ be the nth Leonardo Pisano hybrinomial. The recurrence relation of $L e_{n}^{[H]}(x)$ is

$$
L e_{n}^{[H]}(x)=2 x L e_{n-1}^{[H]}(x)-L e_{n-3}^{[H]}(x) .
$$

Proof. Using equation (6);

$$
\begin{aligned}
L e_{n}^{[H]}(x)= & L e_{n}(x)+\mathbf{i} L e_{n+1}(x)+\varepsilon L e_{n+2}(x)+\mathbf{h} L e_{n+3}(x) \\
= & 2 x L e_{n-1}(x)-L e_{n-3}(x)+\mathbf{i}\left\{2 x L e_{n}(x)-L e_{n-2}(x)\right\} \\
& +\varepsilon\left\{2 x L e_{n+1}(x)-L e_{n-1}(x)\right\}+\mathbf{h}\left\{2 x L e_{n+2}(x)-L e_{n}(x)\right\} \\
= & 2 x L e_{n-1}^{[H]}(x)-L e_{n-3}^{[H]}(x) .
\end{aligned}
$$

Theorem 3. Let $L e_{n}(x)$ be the $n$th Leonardo Pisano polynomial. The generating function of $L e_{n}(x)$ is defined as

$$
g_{L e_{n}(x)}(\lambda)=\sum_{n=0}^{\infty} L e_{n}(x) \lambda^{n}=\frac{1+(1-2 x) \lambda+(2-x) \lambda^{2}}{1-2 x \lambda+\lambda^{3}} .
$$

Proof. Assume that the generating function of Leonardo Pisano polynomial series $\left\{L e_{n}(x)\right\}$ is,

$$
g_{L e_{n}(x)}(\lambda)=\sum_{n=0}^{\infty} L e_{n}(x) \lambda^{n}=L e_{0}(x)+L e_{1}(x) \lambda+L e_{2}(x) \lambda^{2}+\cdots
$$

Using this form, we obtain

$$
\begin{gathered}
-2 x \lambda g_{L e_{n}(x)}(\lambda)=-2 x \lambda L e_{0}(x)-2 x \lambda^{2} L e_{1}(x)-2 x \lambda^{3} L e_{2}(x)-\cdots, \\
\lambda^{3} g_{L e_{n}(x)}(\lambda)=\lambda^{3} L e_{0}(x)+\lambda^{4} L e_{1}(x)+\lambda^{5} L e_{2}(x)+\cdots .
\end{gathered}
$$

If we sum these Equations (11)-(13) side by side, then we obtain

$$
\begin{aligned}
{\left[1-2 x \lambda+\lambda^{3}\right] g_{L e_{n}(x)}(\lambda) } & =L e_{0}(x)+L e_{1}(x) \lambda+L e_{2}(x) \lambda^{2}-2 x L e_{0}(x) \lambda-2 x L e_{1}(x) \lambda^{2} \\
& =L e_{0}(x)+\lambda\left(L e_{1}(x)-2 x L e_{0}(x)\right)+\lambda^{2}\left(\operatorname{Le}_{2}(x)-2 x L e_{1}(x)\right) \\
& =1+(1-2 x) \lambda+(2-x) \lambda^{2}
\end{aligned}
$$

Then the proof is completed. 
Corollary 1. Let $L e_{n}^{[H]}(x)$ be the nth Leonardo Pisano hybrinomial. The generating function of $L e_{n}^{[H]}(x)$ is

$$
G_{L e_{n}^{[H]}(x)}(\lambda)=\frac{L e_{0}^{[H]}(x)+\lambda\left[L e_{1}^{[H]}(x)-2 x L e_{0}^{[H]}(x)\right]+\lambda^{2}\left[L e_{2}^{[H]}(x)-2 x L e_{1}^{[H]}(x)\right]}{1-2 x \lambda^{2}+\lambda^{3}}
$$

Theorem 4. Let $\operatorname{Le}_{n}(x)$ be the nth Leonardo Pisano polynomial. The Binet-like formula for the sequence $\left\{\operatorname{Le}_{n}(x)\right\}$ is

$$
L e_{n}(x)=A \alpha^{n}+B \beta^{n}+C \gamma^{n}
$$

where $\alpha, \beta$ and $\gamma$ are the roots of the characteristic equation $\lambda^{3}-2 x \lambda^{2}+1=0$ and $A, B, C$ are as follows:

$$
\begin{aligned}
& A=\frac{\alpha^{2}+(2-x) \alpha+(x+2)}{(\alpha-\beta)(\alpha-\gamma)}, \\
& B=\frac{\beta^{2}+(2-x) \beta+(x+2)}{(\beta-\alpha)(\beta-\gamma)}, \\
& C=\frac{\gamma^{2}+(2-x) \gamma+(x+2)}{(\gamma-\alpha)(\gamma-\beta)} .
\end{aligned}
$$

Proof. Let $f(\lambda)=\lambda^{3}-2 x \lambda^{2}+1=0$ be the characteristic equation of the recurrence relation $L e_{n}(x)=2 x L e_{n-1}(x)-L e_{n-3}(x)$. It is obvious that $f(\lambda)$ should have three distinct roots $\alpha, \beta$ and $\gamma$. Then, $\frac{1}{\alpha}, \frac{1}{\beta}$ and $\frac{1}{\gamma}$ are the roots of the equation

$$
\begin{aligned}
f(\lambda) & =\lambda^{3}-2 x \lambda^{2}+1=0 \\
f\left(\frac{1}{\lambda}\right) & =\frac{1}{\lambda^{3}}-\frac{2 x}{\lambda^{2}}+1=\frac{\lambda^{3}-2 x \lambda+1}{\lambda^{3}}=0
\end{aligned}
$$

Since $\lambda^{3} \neq 0$, then $h(\lambda)=\lambda^{3}-2 x \lambda+1$.

From the generating function of Leonardo Pisano polynomials and from the sum of geometric series, we have

$$
\begin{aligned}
g_{L e_{n}(x)}(\lambda) & =\frac{1+(1-2 x) \lambda+(2-x) \lambda^{2}}{1-2 x \lambda+\lambda^{3}}=\frac{A}{1-\alpha \lambda}+\frac{B}{1-\beta \lambda}+\frac{C}{1-\gamma \lambda} \\
& =A \sum_{n=0}^{\infty}(\alpha \lambda)^{n}+B \sum_{n=0}^{\infty}(\beta \lambda)^{n}+C \sum_{n=0}^{\infty}(\gamma \lambda)^{n} \\
& =\frac{A(1-\beta \alpha)(1-\gamma \lambda)+B(1-\alpha \lambda)(1-\gamma \lambda)+C(1-\alpha \lambda)(1-\beta \lambda)}{(1-\alpha \lambda)(1-\beta \lambda)(1-\gamma \lambda)}
\end{aligned}
$$

Then we have the following equality

$$
1+(1-2 x) \lambda+(2-x) \lambda^{2}=A(1-\beta \alpha)(1-\gamma \lambda)+B(1-\alpha \lambda)(1-\gamma \lambda)+C(1-\alpha \lambda)(1-\beta \lambda) .
$$

If we substitute $\lambda$ by $\frac{1}{\alpha}$, we obtain

$$
\begin{aligned}
1+(1-2 x) \frac{1}{\alpha}+(2-x) \frac{1}{\alpha^{2}} & =A\left(1-\frac{\beta}{\alpha}\right)\left(1-\frac{\gamma}{\alpha}\right) \\
\alpha^{2}+(1-2 x) \alpha+(2-x) & =A(\alpha-\beta)(\alpha-\gamma)
\end{aligned}
$$

then it is easy to obtain

$$
A=\frac{\alpha^{2}+(1-2 x) \alpha+(2-x)}{(\alpha-\beta)(\alpha-\gamma)}
$$


In the same way, we can obtain $B$ and $C$ as follows:

$$
B=\frac{\beta^{2}+(1-2 x) \beta+(2-x)}{(\beta-\alpha)(\beta-\gamma)}, \quad C=\frac{\gamma^{2}+(1-2 x) \gamma+(2-x)}{(\gamma-\alpha)(\gamma-\beta)} .
$$

If we substitute $A, B, C$ into (20), we obtain the following equation

$$
\begin{aligned}
g_{L e_{n}(x)}(\lambda) & =A \sum_{n=0}^{\infty}(\alpha \lambda)^{n}+B \sum_{n=0}^{\infty}(\beta \lambda)^{n}+C \sum_{n=0}^{\infty}(\gamma \lambda)^{n} \\
& =\sum_{n=0}^{\infty}\left[A \alpha^{n}+B \beta^{n}+C \gamma^{n}\right] \lambda^{n}
\end{aligned}
$$

Consequently, we get

$$
L e_{n}(x)=A \alpha^{n}+B \beta^{n}+C \gamma^{n}
$$

Theorem 5. The Binet-like formula for the Leonardo Pisano hybrinomial sequence is

$$
L e_{n}^{[H]}(x)=A \underline{\alpha} \alpha^{n}+B \underline{\beta} \beta^{n}+C \underline{\gamma} \gamma^{n}
$$

where $\underline{\alpha}=1+\alpha \mathbf{i}+\alpha^{2} \varepsilon+\alpha^{3} \mathbf{h}, \beta=1+\beta \mathbf{i}+\beta^{2} \varepsilon+\beta^{3} \mathbf{h}$ and $\underline{\gamma}=1+\gamma \mathbf{i}+\gamma^{2} \varepsilon+\gamma^{3} \mathbf{h}$. Additionally, $A, B$ and $C$ are as in (17).

Proof. We have $L e_{n}^{[H]}(x)=L e_{n}(x)+\mathbf{i} L e_{n+1}(x)+\varepsilon L e_{n+2}(x)+\mathbf{h} L e_{n+3}(x)$ from (6) and $L e_{n}(x)=A \alpha^{n}+B \beta^{n}+C \gamma^{n}$ from the previous theorem.

$$
\begin{aligned}
L e_{n}^{[H]}(x) & =\left(A \alpha^{n}+B \beta^{n}+C \gamma^{n}\right)+\left(A \alpha^{n+1}+B \beta^{n+1}+C \gamma^{n+1}\right) \mathbf{i} \\
& +\left(A \alpha^{n+2}+B \beta^{n+2}+C \gamma^{n+2}\right) \varepsilon+\left(A \alpha^{n+3}+B \beta^{n+3}+C \gamma^{n+3}\right) \mathbf{h} \\
& =A \underline{\alpha} \alpha^{n}+B \underline{\beta} \beta^{n}+C \underline{\gamma} \gamma^{n} .
\end{aligned}
$$

\section{Some Identities for Leonardo Pisano Polynomial and Hybrinomial Sequences}

In this section we state some identities, such as Cassini-like, d'Ocagne-like identities, where Leonardo Pisano polynomials and hybrinomials are involved.

Theorem 6 (Sum identities). Let $n \geq 1$ be an integer, $\left\{L e_{k}(x)\right\}$ is the Leonardo Pisano polynomial sequence and $\left\{L e_{k}^{[H]}(x)\right\}$ is the Leonardo Pisano hybrinomial sequence, respectively. Then

$$
\begin{aligned}
\text { i. } \quad \sum_{k=0}^{n} L e_{k}(x)= & \frac{1}{2-2 x}\left(4-3 x+L e_{n-1}(x)+L e_{n}(x)-L e_{n+1}(x)\right), \\
\text { ii. } \quad \sum_{k=0}^{n} L e_{k}^{[H]}(x)= & \frac{1}{2-2 x}\left(L e_{0}^{[H]}(x)+L e_{1}^{[H]}(x)+L e_{2}^{[H]}(x)-2 x L e_{0}^{[H]}(x)\right. \\
& \left.-2 x L e_{1}^{[H]}(x)+L e_{n-1}^{[H]}(x)+L e_{n}^{[H]}(x)-L e_{n+1}^{[H]}(x)\right) .
\end{aligned}
$$

\section{Proof.}

i. From the definition of Leonardo Pisano polynomial sequence

$$
L e_{n}(x)=2 x L e_{n-1}(x)-L e_{n-3}(x)
$$


we have the following equations

$$
\begin{array}{cc}
L e_{3}(x) & =2 x L e_{2}(x)-L e_{0}(x) \\
L e_{4}(x) & =2 x L e_{3}(x)-L e_{1}(x) \\
L e_{5}(x) & =2 x L e_{4}(x)-L e_{2}(x) \\
\vdots & \vdots \\
L e_{n}(x) & =2 x L e_{n-1}(x)-L e_{n-3}(x) .
\end{array}
$$

when we sum up these equations side by side we get

$$
\sum_{k=3}^{n} L e_{k}(x)=2 x \sum_{k=2}^{n-1} L e_{k}(x)-\sum_{k=0}^{n-3} L e_{k}(x)
$$

In order to equalize the indexes, we add $P_{1}=L e_{0}(x)+L e_{1}(x)+L e_{2}(x), P_{2}=$ $2 x\left(L e_{0}(x)+L e_{1}(x)+L e_{n}(x)\right)$, and $P_{3}=-\left(L e_{n-2}(x)+L e_{n-1}(x)+L e_{n}(x)\right)$ to both sides. Therefore we can write

$$
P_{2}+P_{3}+\sum_{k=0}^{n} L e_{k}(x)=P_{1}+2 x \sum_{k=0}^{n} L e_{k}(x)-\sum_{k=0}^{n} L e_{k}(x)
$$

Eventually, we obtain the desired result.

ii. The proof can be demonstrated the same way.

We can verify the previous theorem with the following example.

Example 1. The sum of the first four Leonardo Pisano polynomials $L e_{0}=1, L e_{1}=1, L e_{2}=x+2$, $\mathrm{Le}_{3}=2 x^{2}+4 x-1$ is $2 x^{2}+5 x+3$. Indeed, we can verify this result using the previous theorem as follows:

$$
\begin{aligned}
\sum_{k=0}^{3} L e_{k}(x) & =\frac{4-3 x+x+2+2 x^{2}+4 x-1-4 x^{3}-8 x^{2}+2 x+1}{2-2 x} \\
& =\frac{-4 x^{3}-6 x^{2}+4 x+6}{2-2 x}=2 x^{2}+5 x+3 .
\end{aligned}
$$

Theorem 7 (Catalan-like Identities). Let $n$ and $r$ be two non-negative integers and $n \geq r$.

i. The Catalan-like identity for Leonardo Pisano polynomials is

$$
\begin{aligned}
L e_{n+r}(x) L e_{n-r}(x)-L e_{n}^{2}(x)= & A B \alpha^{n-r} \beta^{n-r}\left(\alpha^{r}-\beta^{r}\right)^{2} \\
& +A C \alpha^{n-r} \gamma^{n-r}\left(\alpha^{r}-\gamma^{r}\right)^{2} \\
& +B C \beta^{n-r} \gamma^{n-r}\left(\beta^{r}-\gamma^{r}\right)^{2} .
\end{aligned}
$$

ii. The Catalan-like identity for Leonardo Pisano hybrinomials is

$$
\begin{aligned}
L e_{n+r}^{[H]}(x) L e_{n-r}^{[H]}(x)-\left(L e_{n}^{[H]}(x)\right)^{2}= & A^{2} \alpha^{2 n} \underline{\alpha}^{2}+B^{2} \beta^{2 n} \underline{\beta}^{2}+C^{2} \gamma^{2 n} \underline{\gamma}^{2} \\
& +A B \alpha^{n-r} \beta^{n-r}\left(\alpha^{2 r} \underline{\alpha} \underline{\beta}+\beta^{2 r} \underline{\beta} \underline{\alpha}\right) \\
& +A C \alpha^{n-r} \gamma^{n-r}\left(\alpha^{2 r} \underline{\alpha} \underline{\underline{\gamma}}+\gamma^{2 r} \underline{\gamma} \underline{\alpha}\right) \\
& +B C \beta^{n-r} \gamma^{n-r}\left(\beta^{2 r} \underline{\beta} \underline{\gamma}+\gamma^{2 r} \underline{\gamma}\right) .
\end{aligned}
$$

Proof. Proofs can be easily seen if the Binet-like formula is used for Leonardo Pisano polynomials and Leonardo Pisano hybrinomials. 
Corollary 2 (Cassini-like identities). Let $n$ be a positive integer.

i. The Cassini-like identity for Leonardo Pisano polynomials is

$$
\begin{aligned}
L e_{n+1}(x) L e_{n-1}(x)-L e_{n}^{2}(x) & =A B \alpha^{n-1} \beta^{n-1}(\alpha-\beta)^{2} \\
& +A C \alpha^{n-1} \gamma^{n-1}(\alpha-\gamma)^{2} \\
& +B C \beta^{n-1} \gamma^{n-1}(\beta-\gamma)^{2}
\end{aligned}
$$

ii. The Cassini-like identity for Leonardo Pisano hybrinomials is

$$
\begin{aligned}
L e_{n+1}^{[H]}(x) L e_{n-1}^{[H]}(x)-\left(L e_{n}^{[H]}(x)\right)^{2} & =A^{2} \alpha^{2 n} \underline{\alpha}^{2}+B^{2} \beta^{2 n} \underline{\beta}^{2}+C^{2} \gamma^{2 n} \underline{\gamma}^{2} \\
& +A B \alpha^{n-1} \beta^{n-1}\left(\alpha^{2} \underline{\alpha} \underline{\beta}+\beta^{2} \underline{\beta} \underline{\alpha}\right) \\
& +A C \alpha^{n-1} \gamma^{n-1}\left(\alpha^{2} \underline{\alpha} \underline{\gamma}+\gamma^{2} \underline{\gamma} \underline{\alpha}\right) \\
& +B C \beta^{n-1} \gamma^{n-1}\left(\beta^{2} \underline{\beta} \underline{\gamma}+\gamma^{2} \underline{\gamma} \underline{\beta}\right) .
\end{aligned}
$$

Theorem 8 (d'Ocagne-like identities). Let $m$ and $n$ be two non-negative integers.

i. The d'Ocagne-like identity for Leonardo Pisano polynomials is

$$
\begin{aligned}
L e_{m}(x) L e_{n+1}(x)-L e_{m+1}(x) L e_{n}(x)= & A B(\alpha-\beta)\left(\alpha^{n} \beta^{m}-\alpha^{m} \beta^{n}\right) \\
& +A C(\alpha-\gamma)\left(\alpha^{n} \gamma^{m}-\alpha^{m} \gamma^{n}\right) \\
& +B C(\beta-\gamma)\left(\beta^{n} \gamma^{m}-\beta^{m} \gamma^{n}\right) .
\end{aligned}
$$

ii. The d'Ocagne-like identity for Leonardo Pisano hybrinomials is

$$
\begin{aligned}
L e_{m}^{[H]}(x) L e_{n+1}^{[H]}(x)-L e_{m+1}^{[H]}(x) L e_{n}^{[H]}(x) & =A B(\alpha-\beta)\left(\alpha^{n} \beta^{m} \underline{\alpha} \underline{\beta}-\alpha^{m} \beta^{n} \underline{\beta} \underline{\alpha}\right) \\
& +A C(\alpha-\gamma)\left(\alpha^{n} \gamma^{m} \underline{\alpha} \underline{\gamma}-\alpha^{m} \gamma^{n} \underline{\gamma} \underline{\alpha}\right) \\
& +B C(\beta-\gamma)\left(\beta^{n} \gamma^{m} \underline{\beta} \underline{\gamma}-\beta^{m} \gamma^{n} \underline{\gamma} \underline{\beta}\right)
\end{aligned}
$$

Proof. i. Let us use the Binet-like formula for Leonardo Pisano polynomials.

$$
\begin{aligned}
& L e_{m}(x) L e_{n+1}(x)-L e_{m+1}(x) L e_{n}(x) \\
&= A B \alpha^{m} \beta^{n}(\beta-\alpha)+A C \alpha^{m} \gamma^{n}(\gamma-\alpha)+A B \alpha^{n} \beta^{m}(\alpha-\beta)+B C \beta^{m} \gamma^{n}(\gamma-\beta) \\
&+A C \alpha^{n} \gamma^{m}(\alpha-\gamma)+B C \beta^{n} \gamma^{m}(\beta-\gamma) \\
&= A B(\alpha-\beta)\left(\alpha^{n} \beta^{m}-\alpha^{m} \beta^{n}\right)+A C(\alpha-\gamma)\left(\alpha^{n} \gamma^{m}-\alpha^{m} \gamma^{n}\right) \\
&+B C(\beta-\gamma)\left(\beta^{n} \gamma^{m}-\beta^{m} \gamma^{n}\right) .
\end{aligned}
$$

ii. This can be proven similarly to (i) using the Binet-like formula for Leonardo Pisano hybrinomials.

Author Contributions: Conceptualization, F.K., A.D. and P.C.; methodology, F.K., A.D. and P.C.; software, F.K. and A.D.; validation, F.K., A.D. and P.C.; formal analysis, F.K. and A.D.; investigation, F.K., A.D. and P.C.; resources, F.K., A.D. and P.C.; data curation, F.K., A.D. and P.C.; writing—original draft preparation, F.K. and A.D.; writing-review and editing, F.K., A.D. and P.C.; visualization, F.K. and A.D.; supervision, P.C.; project administration, P.C. All authors have read and agreed to the published version of the manuscript.

Funding: This research was partially funded by Portuguese Funds through FCT—FUNDAÇÃO PARA A CIẼNCIA E A TECNOLOGIA, within the Projects UIDB/00013/2020, UIDP/00013/2020 and UIDB/00194/2020.

Acknowledgments: The last author is a member of the Research Centre CMAT-UTAD (Polo of Research Centre CMAT-Centre of Mathematics of University of Minho) and also a collaborat- 
ing member of the Research Centre CIDTFF-Research Centre on Didactics and Technology in the Education of Trainers of University of Aveiro.

Conflicts of Interest: The authors declare no conflict of interest.

\section{References}

1. Koshy, T. Fibonacci and Lucas Numbers with Applications; John Wiley and Sons: Hoboken, NJ, USA, 2019.

2. Horadam, A.F. A generalized Fibonacci sequence. Am. Math. Mon. 1961, 68, 455-459. [CrossRef]

3. Horadam, A.F. Basic properties of a certain generalized sequence of numbers. Fibonacci Q. 1965, 3, $161-176$.

4. Halici, S. On Fibonacci quaternions. Adv. Appl. Clifford Algebras 2012, 22, 321-327. [CrossRef]

5. Dağdeviren, A.; Kürüz, F. On The Horadam Hybrid Quaternions. arXiv 2020, arXiv:2012.08277.

6. Catarino, P.; Borges A. On Leonardo numbers. Acta Math. Univ. Comenianae 2019, 89, 75-86.

7. Shannon, A.G. A note on generalized Leonardo numbers. Notes Number Theory Discrete Math. 2019, 25, 97-101. [CrossRef]

8. Catarino, P.; Borges, A. A note on incomplete Leonardo numbers. Integers 2020, 20, 1-7.

9. Alp, Y.; Koçer, E.G. Some properties of Leonardo numbers. Konuralp J. Math. 2021, 9, 183-189.

10. Alp, Y.; Koçer, E.G. Hybrid Leonardo numbers. Chaos Solitons Fractals 2021, 150, 111-128. [CrossRef]

11. Özdemir, M. Introduction to hybrid numbers. Adv. Appl. Clifford Algebras 2018, 28, 1-32. [CrossRef]

12. Bicknell, M. A primer for the Fibonacci numbers VII. Fibonacci Q. 1970, 8, 407-420.

13. Horzum, T.; Kocer, E.G. On some properties of Horadam polynomials. Int. Math. Forum 2009, 25, $1243-1252$.

14. Hoggatt, V.E., Jr.; Bicknell, M. Roots of Fibonacci polynomials. Fibonacci Q. 1973, 11, 271-274.

15. Catarino, P. The h(x)-Fibonacci quaternion polynomials: Some combinatorial properties. Adv. Appl. Clifford Algebras 2016, 26, 71-79. [CrossRef]

16. Özkan, E.; Altun, İ. Generalized Lucas polynomials and relationships between the Fibonacci polynomials and Lucas polynomials. Commun. Algebra 2019, 47, 4020-4030. [CrossRef]

17. Liana, M.; Szynal-Liana, A.; Wloch, I. On Pell hybrinomials. Miskolc Math. Notes 2019, 20, 1051-1062. [CrossRef]

18. Szynal-Liana, A.; Włoch, I. Introduction to Fibonacci and Lucas hybrinomials. Complex Var. Elliptic Equ. 2020, 65, $1736-1747$. [CrossRef]

19. Petroudi, S.H.J.; Pirouz, M. On Some Properties and Identities of Van Der Laan Hybrinomail Sequence, Preprint. In Proceedings of the 5th International Conference on Combinatorics, Cryptography, Computer Science and Computing, Tehran, Iran, 17-18 November 2021.

20. Petroudi, S.H.J.; Pirouz, M.; Ozkoc, A. The Narayana Polynomial and Narayana Hybrinomial Sequences. Konuralp J. Math. 2021 9, 90-99.

21. Kizilateş, C. A Note on Horadam Hybrinomials. Preprints 2020. [CrossRef] 